\title{
Weight-Based Adaptation of TNF-Antagonist Induction versus Maintenance Dose
}

\author{
Alexander A. Navarini ${ }^{a}$ Marco A. Muster ${ }^{\mathrm{a}}$ \\ Antonios G.A. Kolios ${ }^{\mathrm{a}}$ Philipp Fritsche ${ }^{\mathrm{a}}$ Martin Glatz ${ }^{\mathrm{a}}$ \\ Lars E. French ${ }^{a}$ Ralph M. Trüeb ${ }^{a, b}$ \\ ${ }^{a}$ Department of Dermatology, University Hospital of Zurich, Zurich, and bSkin and \\ Hair Center Professor Trüeb, Wallisellen, Switzerland
}

\section{Key Words}

Psoriasis vulgaris · Obesity · Pharmacology · TNF-alpha · Adalimumab

\begin{abstract}
Biologics are highly specific and exhibit few problems in regard to overdosages. In clinical practice, induction schemes with an initial loading dose and a subsequent lower maintenance dose have been established and are of higher efficacy for psoriasis than starting directly with the maintenance dose. As obese patients sometimes respond less well to standard dosages, increases of the maintenance dose, but not the loading doses, have been tried with variable success. In our study, we increased the loading (160 mg instead of $80 \mathrm{mg}$ ) but not the maintenance dose of adalimumab in an obese patient with severe psoriasis resistant to previous biologics and methotrexate. Within 12 weeks, both PASI (11 to 1.6$)$ and DLQI (22/30 to 5/30) decreased. This strategy might be an effective and less costly alternative to doubling the maintenance doses, and could be further evaluated for psoriasis patients refractory to previous treatments.
\end{abstract}

\section{Introduction}

Biologic agents specifically target cytokines or surface structures to counteract pathogenic immune activation. Due to their high specificity, they usually neither exhibit organ toxicities nor inhibit other processes or interact with other drugs, even in high dosages. Apart from the standard dose recommendations, as determined in phase II and III studies, our knowledge of alternate dosing for higher efficacy in special situations is still scarce. Obesity is a common factor held responsible for compromised efficacy of fixed dosed biologics such as etanercept versus weight-based dosed medication such as infliximab, probably due to a higher distribution volume with decreased concentrations of 
the biologic agent [1]. Adalimumab in a dosage of $40 \mathrm{mg}$ weekly versus biweekly has been shown to have an increased efficacy in patients with psoriasis previously treated with other biologics [2]. Alternatively, some experts have used doubled maintenance dosages of adalimumab $80 \mathrm{mg}$ biweekly for obese patients [pers. comm.]. However, this strategy is very costly and, in our hands, has not proven particularly effective, although systematic studies are lacking. However, because these drugs target dynamic immunologic processes, modification of doses at critical time points, e.g. at the beginning of therapy, might yield higher efficacy than elevation of maintenance doses. Hence, we increased the induction dose instead of the maintenance dose of adalimumab in an obese patient with severe psoriasis previously treated with other biologics.

\section{Case Presentation}

A 49-year-old Caucasian male with obesity (BMI 34.4) and arterial hypertension presented with a 32-year history of severe plaque-type psoriasis associated since 3 years with psoriatic arthritis. Previously, the HLA-Cw6-positive patient had initially been successfully treated with the weight-based dosed efalizumab, but in 2007, he was eventually switched to etanercept $2 \times 25 \mathrm{mg}$ weekly because of the development of psoriatic arthritis. Although a decrease of the arthritis pain was noted, the PASI response was not satisfactory and the patient gained $12 \mathrm{~kg}$ in weight. Instead of etanercept, methotrexate $15 \mathrm{mg}$ s.c. once a week was started; but PASI increased further to a score of 13.1 within 2 months. At this time point, infliximab $5 \mathrm{mg} / \mathrm{kg}$ body weight i.v. was introduced and combined with methotrexate $7.5 \mathrm{mg}$; however, PASI50 was not reached after 12 weeks, and infliximab was stopped again.

Based on the patient's wish, we restarted efalizumab with subsequent reduction of PASI down to 2.1 until April 2009. However, as efalizumab was drawn back from the market due to reports of progressive multifocal leukoencephalopathy, a change of treatment was required. Therefore, in April 2009 ustekinumab was started at a dosage of $90 \mathrm{mg}$ s.c., as recommended for patients heavier than $100 \mathrm{~kg}$. The patient's PASI increased from 2.1 to 11 ( $\underline{\text { fig. } 1}$ ) within 14 weeks, and thus his impairment of quality of life reached a level of desperation, and he injected $15 \mathrm{mg}$ methotrexate at week 14 on his own initiative. Finally, we considered a switch to adalimumab. For this agent, the induction dose seems to be especially important, as patients started on $40 \mathrm{mg}$ s.c., such as for treatment of psoriatic arthritis, show inferior 12-week PASI75 responses [3,4] to induction doses of $80 \mathrm{mg}$ s.c. [5]. However, weight-adapted treatment protocols in regard to the induction dose do not exist yet. Therefore, we started a therapy with adalimumab with a doubled loading dose of $160 \mathrm{mg}$ in August 2009 (fig. 2), followed by a second dose of $80 \mathrm{mg}$ s.c. two weeks later, and continued with the normal maintenance dose of $40 \mathrm{mg}$ s.c. biweekly. This dosing protocol is effective for the treatment of Crohn's disease [6]. Within the following 12 weeks, both PASI and DLQI decreased significantly (fig. 3 ) (11 to 1.6 and 22/30 to 5/30, respectively).

\section{Comment/Discussion}

In clinical practice, fixed dosing of biologic agents as opposed to weight-based dosing seems to be relevant, especially in overweight patients. The suspicion that a drug dose optimized for the typical 70-kg patient might be insufficient for a $120-\mathrm{kg}$ person, for example, is obvious, and has been shown for etanercept [7] and alefacept [8], but is not yet clearly proven for adalimumab nor ustekinumab. Although a consensus might be easily found that biologics must be adequately dosed in special situations, such as in obese patients or in patients previously treated with other biologics, it is not clear whether this is crucial during the induction phase or in the maintenance phase of therapy. Because pathologic immune responses can be self-perpetuating processes $[9,10]$, it could be a sensible strategy to not only dampen the target cytokine with biologics, but also try to inhibit it as much as possible, especially at the beginning of therapy. Accordingly, 
strategies with an early higher dose followed by lower maintenance doses have made their way in daily clinical protocols such as for adalimumab or infliximab. However, if early inhibition is more important than the maintenance dose, then we might be better advised to increase the starting dose in obese patients rather than the maintenance dose, such as in our indicator patient, in the hope that the pathologic immune response might be terminated early on.

Biologics are not easily overdosed, as complete inhibition of the target cytokine or surface antigen is the therapeutic goal already at recommended doses. Their immunologic specificity is high, so that even at extreme doses, unwanted inhibition of, i.e. other cytokines, is not to be expected. In addition, in case overdose-induced side effects should occur, e.g. cardiac failure at $10 \mathrm{mg} / \mathrm{kg}$ infliximab [11], it might be better to overdose at the start and expect adverse reactions at the beginning of the therapy rather than run the risk of partly unknown insidious chronic changes that could result from long-term overdosing of biologics. On the contrary, underdosing of biologics might be much more problematic because of the induction of neutralizing antibodies [12] that have also been shown to hamper the long-term efficacy of adalimumab $[13,14]$. A limitation to our observation is that we do not know whether the patient would have had a satisfactory response to the normal loading dose of adalimumab, but on the basis of the patient's history and our experience with other patients with a similar profile of overweight and/or previous biologic therapies, we expected a further treatment failure with adalimumab in the normal dose. In addition, whether nonresponse to the normal induction scheme with subsequent increased induction dose as described here would lead to the same result remains unknown. Taken together, our overweight patient with severe psoriasis and previous biologic therapy had a good clinical response to a doubled loading dose of adalimumab as used for Crohn's disease, while maintenance doses were not altered. This is a strategy that could be further evaluated for obese psoriasis patients refractory to previous treatments, including other biologics.

\section{Disclosure Statement}

The authors have no conflicts of interest to disclose. 


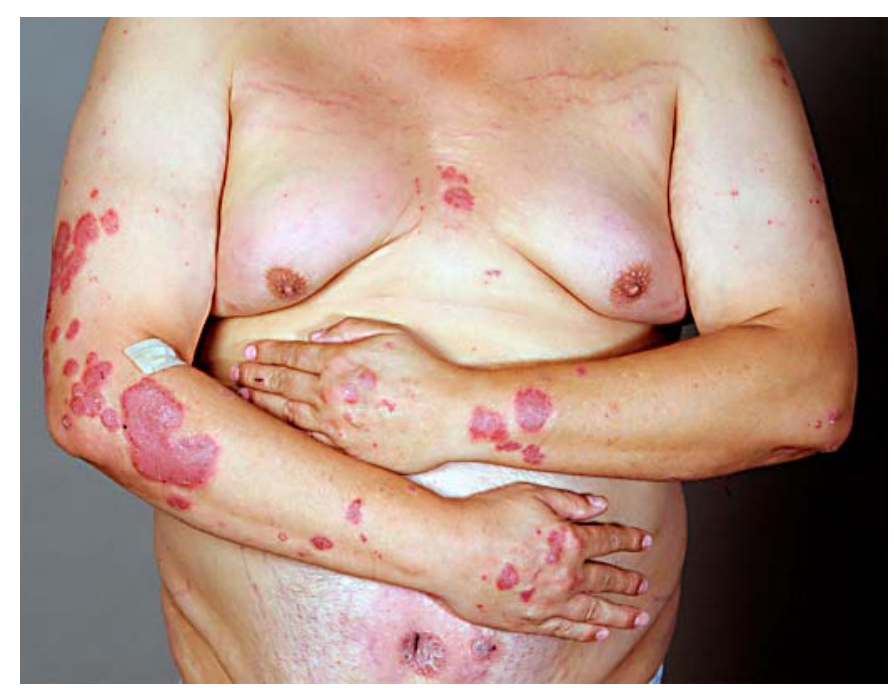

Fig. 1. Patient before treatment with adalimumab.

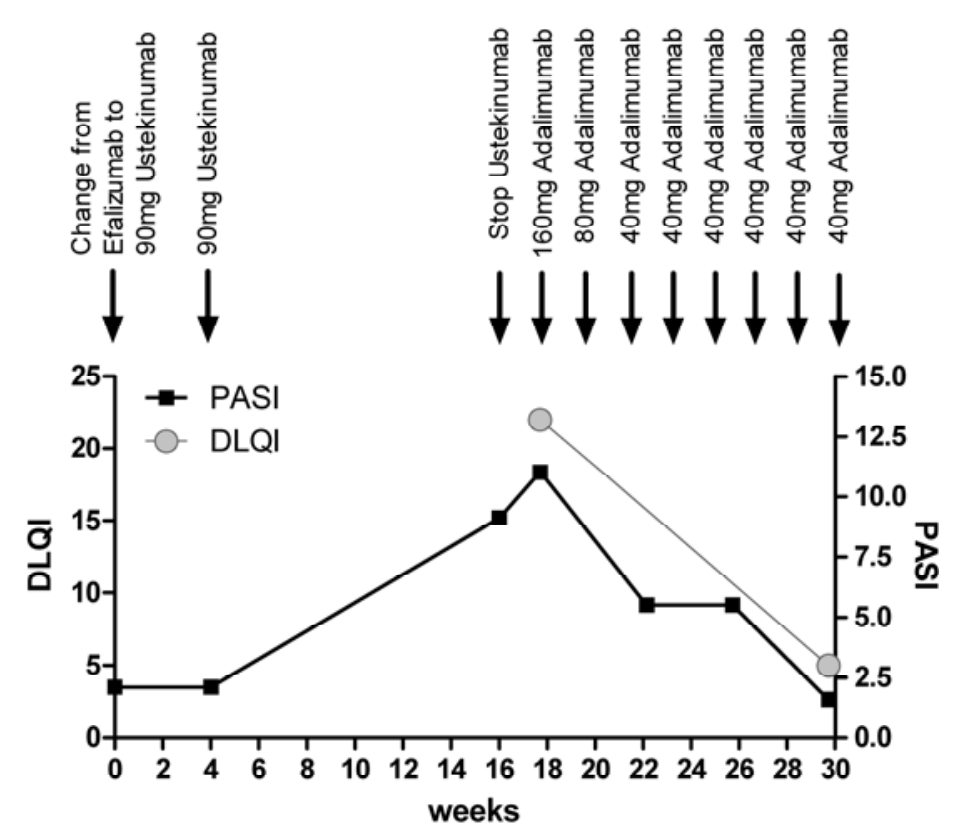

Fig. 2. PASI and DLQI score during treatment with adalimumab. 


\begin{tabular}{|c|c|c|c|}
\hline $\begin{array}{l}\text { Case Reports in } \\
\text { cermatology }\end{array}$ & $\begin{array}{l}\text { Case Rep Dermatol 2011;3:124-129 } \\
\text { DOI: 10.1159/000329343 }\end{array}$ & $\begin{array}{l}\text { Published online: } \\
\text { June } 18,2011\end{array}$ & \begin{tabular}{|l} 
@ 2011 S. Karger AG, Basel \\
ISSN 1662-6567 \\
www.karger.com/cde
\end{tabular} \\
\hline
\end{tabular}

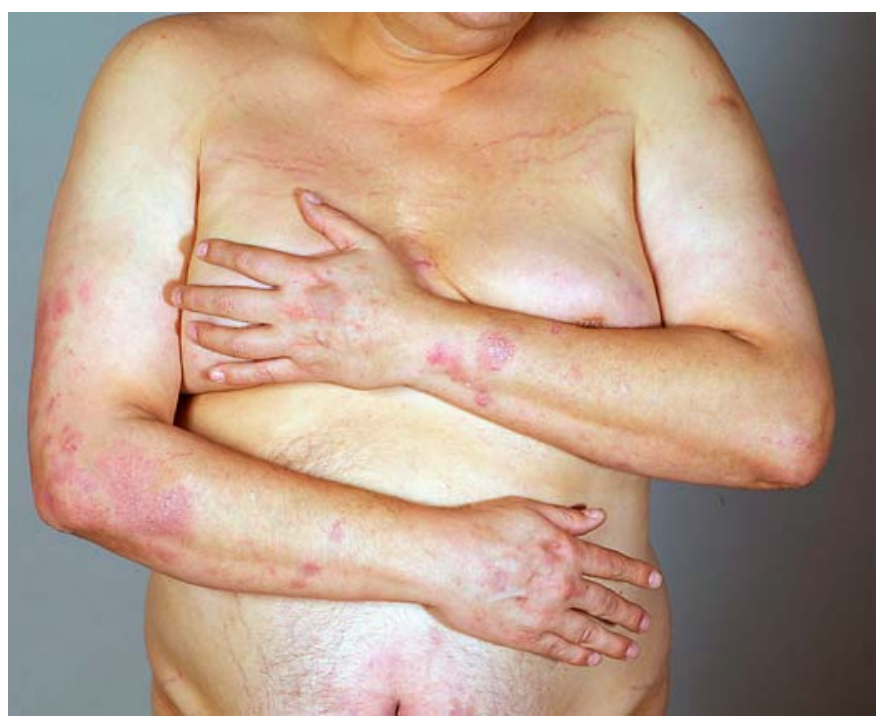

Fig. 3. Patient after 12 weeks of adalimumab with adapted induction dose.

\section{References}

1 Clark L, Lebwohl M: The effect of weight on the efficacy of biologic therapy in patients with psoriasis. J Am Acad Dermatol 2008;58:443-446.

$\checkmark 2$ Papoutsaki M, Chimenti MS, Costanzo A, et al: Adalimumab for severe psoriasis and psoriatic arthritis: an open-label study in 30 patients previously treated with other biologics. J Am Acad Dermatol 2007;57:269-275.

3 Gladman DD, Mease PJ, Ritchlin CT, et al: Adalimumab for long-term treatment of psoriatic arthritis: fortyeight week data from the adalimumab effectiveness in psoriatic arthritis trial. Arthritis Rheum 2007;56:476488.

4 Mease PJ, Ory P, Sharp JT, et al: Adalimumab for long-term treatment of psoriatic arthritis: 2-year data from the Adalimumab Effectiveness in Psoriatic Arthritis Trial (ADEPT). Ann Rheum Dis 2009;68:702-709.

$\checkmark 5$ Menter A, Tyring SK, Gordon K, et al: Adalimumab therapy for moderate to severe psoriasis: A randomized, controlled phase III trial. J Am Acad Dermatol 2008;58:106-115.

-6 Hanauer SB, Sandborn WJ, Rutgeerts P, et al: Human anti-tumor necrosis factor monoclonal antibody (adalimumab) in Crohn's disease: the CLASSIC-I trial. Gastroenterology 2006;130:323-333; quiz 591.

7 Strober B, Gottlieb A, Leonardi C, Papp K: Poster 2890: Levels of response of psoriasis patients with different baseline characteristics treated with etanercept. Journal of the American Academy of Dermatology 2006;54:AB220.

8 Marzella L, Papadopoulos E: Final clinical review: biologic license application STN BL 125036/0 for alefacept for treatment of moderate to severe chronic plaque psoriasis; in Administration $\mathrm{FaD}$ (ed): [internet memorandum], Silver Spring (MD): available at: http://www.fda.gov/ohrms/dockets/ac/02/minutes/3865M1.htm (accessed April 23, 2010;2002).

-9 Bowcock AM, Krueger JG: Getting under the skin: the immunogenetics of psoriasis. Nat Rev Immunol 2005;5:699-711.

10 Kupper TS: Immunologic targets in psoriasis. N Engl J Med 2003;349:1987-1990.

11 Chung ES, Packer M, Lo KH, Fasanmade AA, Willerson JT: Randomized, double-blind, placebo-controlled, pilot trial of infliximab, a chimeric monoclonal antibody to tumor necrosis factor-alpha, in patients with moderate-to-severe heart failure: results of the anti-TNF Therapy Against Congestive Heart Failure (ATTACH) trial. Circulation 2003;107:3133-3140.

12 Gottlieb AB, Evans R, Li S, et al: Infliximab induction therapy for patients with severe plaque-type psoriasis: a randomized, double-blind, placebo-controlled trial. J Am Acad Dermatol 2004;51:534-542.

13 Lecluse LL, Driessen RJ, Spuls PI, et al: Extent and clinical consequences of antibody formation against adalimumab in patients with plaque psoriasis. Arch Dermatol 2010;146:127-132. 
14 van Kuijk AW, de Groot M, Stapel SO, Dijkmans BA, Wolbink GJ, Tak PP: Relationship between the clinical response to adalimumab treatment and serum levels of adalimumab and anti-adalimumab antibodies in patients with psoriatic arthritis. Ann Rheum Dis 2010;69:624-625. 